\title{
Comparison of Different Environmental Conditions in the Follow-up of Subarachnoid Haemorrhage: An Experimental Rat Study
}

\author{
Turan KANDEMIR ${ }^{1}$, Fulya Buge ERGEN ${ }^{4}$, Didem TURGUT COSAN² ${ }^{2}$ Fezan MUTLU ${ }^{3}$, Zuhtu OZBEK ${ }^{1}$ \\ Tevfik Erhan COSAN ${ }^{1}$ \\ ${ }^{1}$ Eskişehir Osmangazi University, Faculty of Medicine, Department of Neurosurgery, Eskisehir, Turkey \\ ${ }^{2}$ Eskişehir Osmangazi University, Faculty of Medicine, Department of Medical Biology, Eskisehir, Turkey \\ ${ }^{3}$ Eskişehir Osmangazi University, Faculty of Medicine, Department of Biostatistics, Eskisehir, Turkey \\ ${ }^{4}$ Eskişehir Osmangazi University, Health Science Institute, Department of Interdisciplinary Neuroscience, Eskisehir, Turkey
}

Corresponding author: Zuhtu OZBEK zuhtuozbek@gmail.com

\section{ABSTRACT}

AIM: To analyze the effect of different environmental conditions on the brain in rats with subarachnoid haemorrhage.

MATERIAL and METHODS: Microtubules in neurons mediate both the consciousness and memory and regulate firing. Microtubuleassociated proteins promote microtubule organisation and dynamics. We investigated MAP2, tau and amyloid beta levels in the hippocampus and the frontal cortex after experimental subarachnoid haemorrhage in rats. Subjects were divided into subgroups and were housed either in an enriched, standard or isolated environment. Tissue levels were measured on day 7 for short-term outcomes and on day 14 for long-term outcomes after subaracnoid haemorrhage.

RESULTS: After subaracnoid haemorrhage, decreased microtubule-associated proteins 2 levels, a trend in pathologic tau accumulation and increased amyloid beta levels in different brain regions of rats kept in an isolated environment. Frontal lobe microtubule-associated proteins 2 levels were increased in rats kept in an enriched environment for 7 days. Pathological hippocampal tau and frontal lobe amyloid beta levels were increased in rats kept in an isolated environment for 7 days. Increased microtubuleassociated proteins 2 levels in the hippocampus, decreased frontal and hippocampal amyloid beta were seen in rats kept in an enriched environment for 14 days.

CONCLUSION: Although it would be too early to offer recommendations, results of the present study support that an enriched environment may be more valuable in the follow-up of subaracnoid haemorrhage. Further experimental studies would provide more reliable results to facilitate discussions about how to optimise the patient's environmental conditions.

KEYWORDS: Subarachnoid haemorrhage, Environmental conditions, Rat, MAP2, Tau, Amyloid beta

\section{INTRODUCTION}

S ubarachnoid haemorrhage (SAH) is a medical emergency associated with high morbidity and mortality. Diffuse brain damage, secondary complications and elevated intracranial pressure may cause functional deficits in consciousness, cognition and memory in addition to impaired clinical status on different scales (1). Many hospitalised patients with SAH need long-term follow-up in the intensive or intermediate care unit. However, there is no definite and defined consensus on whether the nature of a patient's environmental condition has an effect or not. Although neurons of both the hippocampus and cortex are known to be positively affected in an enriched environment $(12,15)$, the overall belief and tendency in the

\begin{tabular}{llll}
\hline Turan KANDEMIR & (D) : 0000-0002-6230-0645 & Fezan MUTLU & (D) : 0000-0002-9339-4031 \\
Fulya Buge ERGEN & (D) : 0000-0001-8089-9033 & Zuhtu OZBEK & (D) : 0000-0003-0028-4181 \\
Didem TURGUT COSAN & (D) : $0000-0002-8488-6405$ & Tevfik Erhan COSAN (D): 0000-0002-9642-6596
\end{tabular}


follow-up of SAH are to keep the patient isolated or in a standard environment.

Microtubules, the major components of neurons, mediate both consciousness and memory and regulate firing (6). Their functions are important in neuronal development, plasticity and polarisation. Disruptions of microtubule organisation and dynamics may cause loss of neuronal integrity and function $(5,8)$. Tau and other microtubule-associated proteins (MAPs) promote both the assembly and stabilisation of neuronal microtubules (5). They regulate microtubule networks and their functions in axons and dendrites of neurons $(4,16)$. The MAP2/tau family of proteins are originally characterised by their ability to bind microtubules. In brain trauma and damage, decreased cortical and hippocampal levels of MAP2 have been shown $(2,10)$. Increased amyloid beta induces pathological changes in tau proteins and neuronal cells. Thus, the combined consideration of amyloid beta and tau proteins is a more reliable indicator to evaluate the pathological accumulation of tau $(3,22,25)$.

We investigated MAP2, tau and amyloid beta levels in the hippocampus and the frontal cortex after experimental subarachnoid haemorrhage in rats housed either in enriched, standard or isolated environments.

\section{MATERIAL and METHODS}

All procedures performed in studies involving animals were in accordance with the ethical standards of the institution or practice at which the studies were conducted [Eskisehir Osmangazi University the Care and Use of Animals Ethical Committee (Approval No: 597/23.05.2017)].

All applicable international, national and/or institutional guidelines for the care and use of animals were followed.

\section{Groups}

One hundred twenty-two Sprague Dawley female rats with a weight of 250-350 g were selected from a group of healthy 2.5-3 month-olds. Rats were first divided into the SAH and the control group, and then these groups were subdivided into different environments. A double-haemorrhage model was used to create the SAH model (tail blood was injected into the cisterna magna). Eighty experimental rats were used for the $\mathrm{SAH}$ model, and 38 of them died during the applied surgery or in the short post-surgical period.

Initially, each animal was held in a single standard cage for one day. Then, 7 rats from each group were placed into previously prepared cages with different environmental conditions. For control groups, another 42 rats were placed in cages with different environmental conditions so that 7 rats would be kept in each group without surgery.

On the condition of being able to examine the effects of different environmental conditions for both short and long periods of time, half of the rats were kept for 7 days and the other half for 14 days. The list of experimental groups is provided in Table I.

\section{Cages for Different Environments}

For the standard environment, standard rat cages were used, and 3-4 rats were placed in each cage. Rats were maintained at normal room temperature, provided 12-hour light and dark cycle conditions and allowed continuous access to food.

Special cages were prepared for an enriched environment. These cages were larger and had two flats with a staircase for the rats to reach the flats. Several toys were left in the cage and were changed every 48 hours. Between the hours 19:00 and 20:00, classical music accompanied by a low-pitched voice was piped into cages. Rats were maintained at normal

Table I: The List of Experimental Groups

\begin{tabular}{ll}
\hline GRoups & \\
\hline 1.Group & Control +7 days in STANDARD environment (SE7) \\
\hline 2.Group & Control +7 days in ISOLATED environment (IE7) \\
\hline 3.Group & Control +7 days in ENRICHED environment (EE7) \\
\hline 4.Group & SAH (1cc autologous blood $)+7$ days in STANDARD environment (SAH SE7) \\
\hline 5.Group & SAH (1cc autologous blood $)+7$ days in ISOLATED environment (SAH IE7) \\
\hline 6.Group & SAH (1cc autologous blood $)+7$ days in ENRICHED environment (SAH EE7) \\
\hline 7.Group & Control +14 days in STANDARD environment (SE14) \\
\hline 8.Group & Control +14 days in ISOLATED environment (IE 14) \\
\hline 9.Group & Control +14 days in ENRICHED environment (EE 14) \\
\hline 10.Group & SAH (1cc autologous blood $)+14$ days in STANDARD environment (SAH SE14) \\
\hline 11.Group & SAH (1cc autologous blood $)+14$ days in ISOLATED environment (SAH IE14) \\
\hline 12.Group & SAH (1cc autologous blood) +14 days in ENRICHED environment (SAH EE14) \\
\hline
\end{tabular}


room temperature, provided 12-hour light and dark cycle conditions and allowed continuous access to food.

For the isolated environment, cages smaller than those used for the standard environment were used. Cages were covered with aluminium foil on all four sides, and with the top open, a cage was prepared for each animal. Rats were maintained at normal room temperature, provided 12-hour light and dark cycle conditions and allowed continuous access to food.

\section{Collection of Tissue Samples}

Rats in the short-term group were anaesthetised on day 7 and rats in the long-term group were anaesthetised on day 14 (intramuscular $5 \mathrm{mg} / \mathrm{kg}$ xylazine hydrochloride, $30 \mathrm{mg} / \mathrm{kg}$ ketamine).

After their aortas were perfused with 200 cc normal saline, rats were decapitated, and brains were immediately removed and placed on ice. The hippocampus and the prefrontal cortex were dissected, frozen and stored at $-80^{\circ} \mathrm{C}$.

Measurement of MAP2, tau protein and amyloid beta levels in tissue samples

At the beginning of the measurement, samples were taken out of $-80{ }^{\circ} \mathrm{C}$, placed in $1 \mathrm{~mL}$ PBS and homogenised with

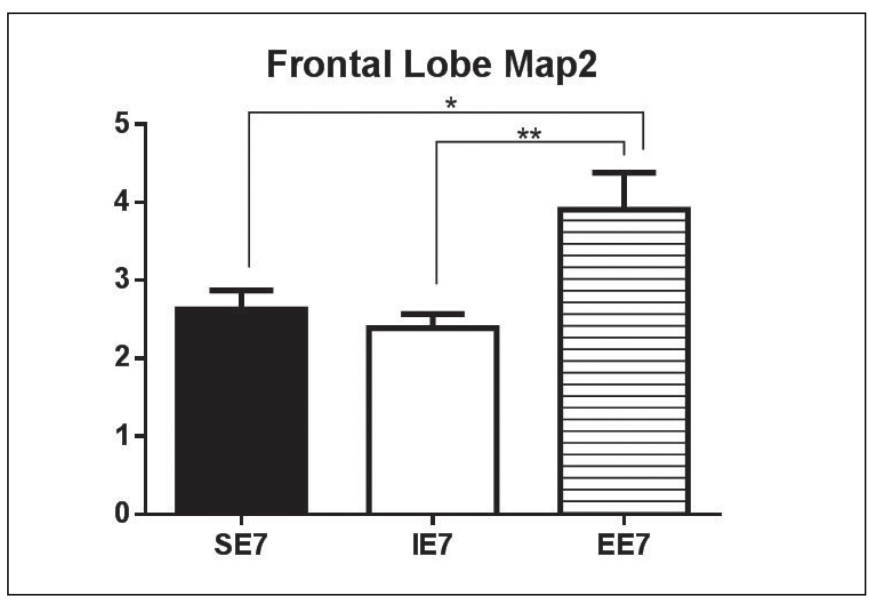

Figure 1: Frontal lobe MAP2 levels (ng/ml) in control group rats kept in different environments for 7 days $\left({ }^{*} p<0,05\right.$, $\left.{ }^{* *} p<0,01\right)$. a knife homogeniser while on ice. Specific ELISA tests were used to identify MAP2, tau protein and amyloid beta levels in the samples.

For MAP2 protein level determinations, 'CUSABIO microtubule-associated protein 2 (MAP2) ELISA Kit (Cat No: CSB-E12806r)'; for $A \beta$ protein level determinations, 'MyBioSourceRat total amyloid beta peptide ELISA Kit (Cat No: MBS728317)'; for Tau protein level determinations, 'Elabscience Rat MAPt (Microtubule Associated Protein Tau/ Tau Protein) ELISA Kit (Cat No: E-EL-R0943)' procedures were performed.

The absorbance values of samples, as indicated in the kit procedures, were determined by an ELISA reader (Multiskan ${ }^{\mathrm{TM}}$ GO Microplate Spectrophotometer, Thermo Scientific, MA, USA) at $450 \mathrm{~nm}$.

\section{Statistical Analysis}

A Shapiro-Wilk normality test was performed for continuous variables. Data with homogeneous variances were analysed using an analysis of variance and Tukey's post-test and were presented as means \pm standard deviations. Nonparametric data were analysed using Kruskal-Wallis test and were presented as median (25\%-75\%) percentiles. SPSS 21.0 (Chicago, IL, USA) was used for statistical analysis. GraphPadPrism 6 was used for graphics, and $p<0.05$ was considered as significant.

\section{RESULTS}

\section{Control Group}

In the control group kept in different environments for 7 days, frontal lobe MAP2 levels of an enriched environment were significantly increased compared with those of other groups (Figure 1). Although there was no statistical difference in tau, both frontal lobe and hippocampal amyloid beta levels statistically increased in rats kept in an isolated environment (Figure 2).

In the control group kept in different environments for 14 days, hippocampal MAP2 levels of an enriched environment were significantly increased compared with those of an isolated environment (Figure 3). Frontal lobe and hippocampal

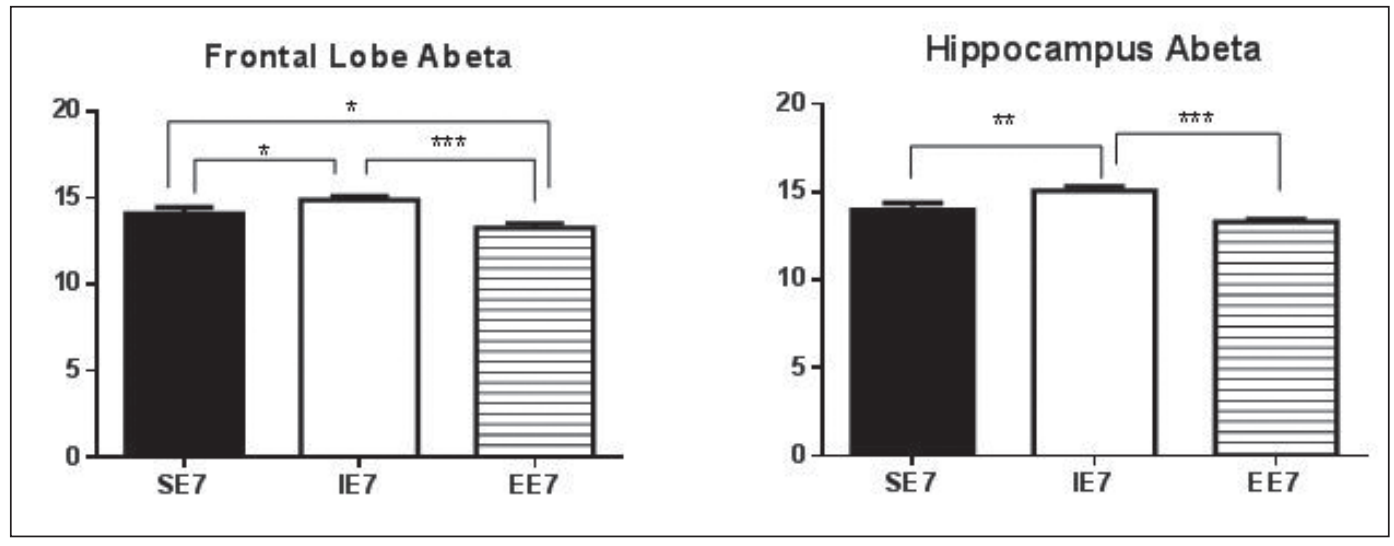

Figure 2: Frontal lobe and hippocampus amyloid beta levels (A beta, $\mathrm{ng} / \mathrm{ml}$ ) in control group rats kept in different environments for 7 days $\left({ }^{*} \mathrm{p}<0.05\right.$, $\left.{ }^{\star *} \mathrm{p}<0.01,{ }^{* * *} \mathrm{p}<0.001\right)$. 
amyloid beta levels statistically increased in rats kept in an isolated environment compared with rats kept in an enriched environment (Figure 4).

\section{MAP2 Levels in SAH Groups}

In the SAH group kept in different environments for 7 days,

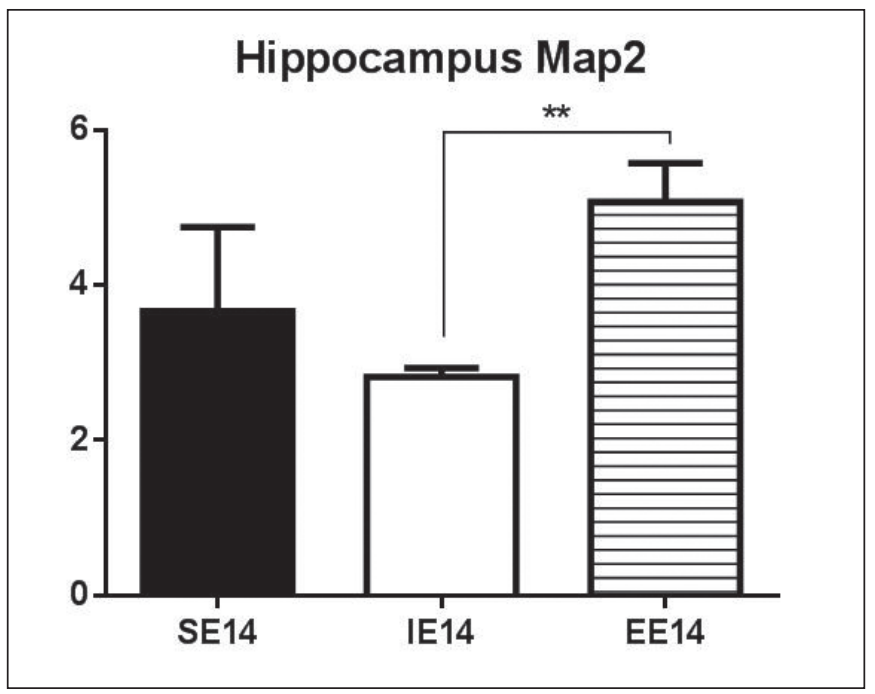

Figure 3: Hippocampus MAP2 levels ( $\mathrm{ng} / \mathrm{ml}$ ) in control group rats kept in different environments for 14 days $\left({ }^{* \star} p<0.01\right)$. rats kept in an enriched environment showed a tendency of increased MAP2 levels in both the frontal cortex and hippocampus but they did not reach the level of statistical significance. The only statistically significant increase was seen in the frontal lobe of rats kept in an enriched environment compared with rats that were kept in an isolated environment (Figure 5).

In the SAH group kept in different environments for 14 days, a statistically significant decrease in hippocampal MAP2 levels was seen in rats kept in an isolated environment compared with those in control rats kept in a standard environment (Figure 6). Although there was a decreased tendency of frontal lobe MAP2 levels in rats kept in an isolated environment, it showed no statistical significance.

When compared with the control group rats kept in a standard environment, frontal lobe MAP2 levels were significantly decreased in the $\mathrm{SAH}$ group rats kept in an isolated environment for 7 days and also decreased in the SAH group rats kept in an isolated environment for 14 days (Figure 7).

\section{Tau and Amyloid beta (A beta) levels in SAH groups}

In the SAH group kept in different environments for 7 days, hippocampal tau levels showed significantly increased accumulation in rats kept in an isolated environment compared with SAH groups kept in other environments, and the control group kept in a standard environment (Figure 8).
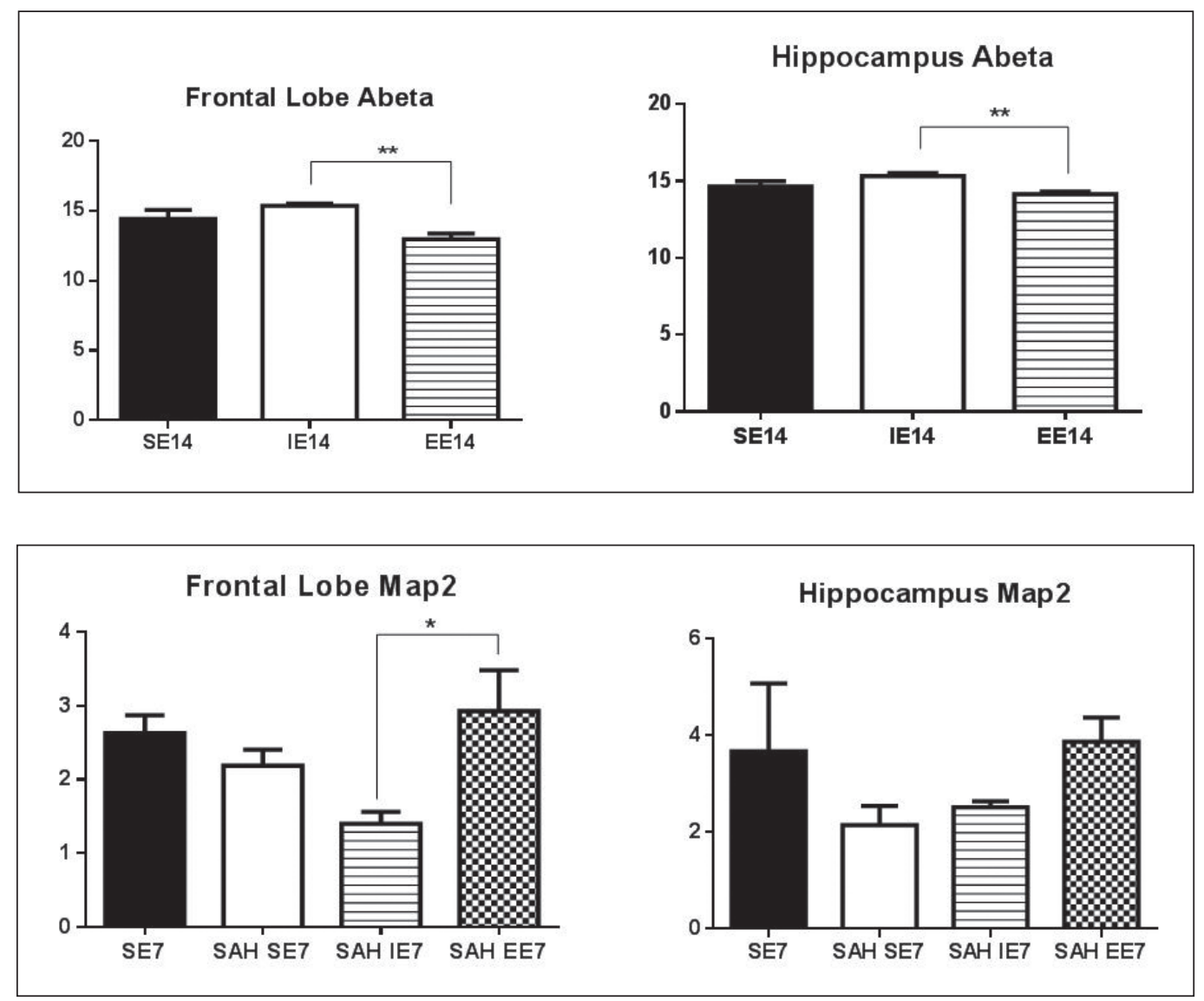

Figure 4: Frontal lobe and hippocampus amyloid beta levels $(\mathrm{ng} / \mathrm{ml})$ in control group rats kept in different environments for 14 days $\left({ }^{\star *} \mathrm{p}<0.01\right)$

Figure 5: Frontal lobe and hippocampus MAP2 levels $(\mathrm{ng} / \mathrm{ml})$ in control and SAH group rats kept in different environments for 7 days $\left({ }^{*} \mathrm{p}<0.05\right)$. 


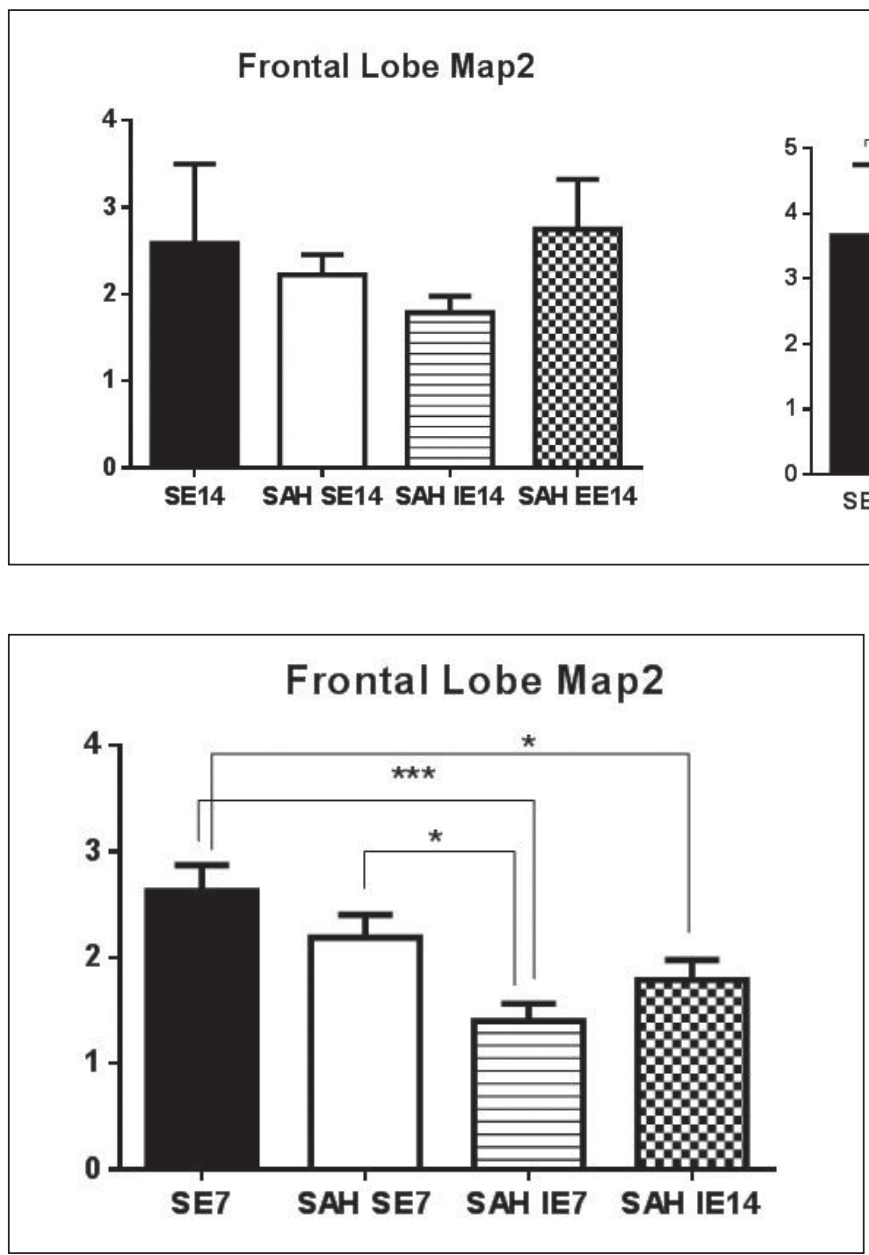

Figure 7: Comparison of frontal lobe MAP2 levels $(\mathrm{ng} / \mathrm{ml})$ in control and SAH group rats kept in different environments for 7 and 14 days $\left({ }^{*} p<0.05,{ }^{* *} p<0.01,{ }^{* * *} p<0.001\right)$.

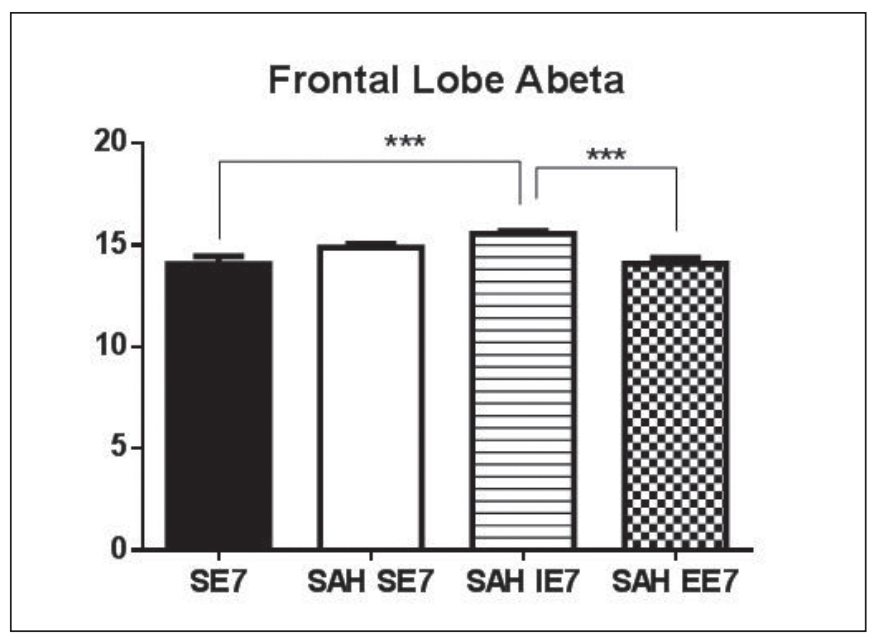

Figure 9: Frontal lobe amyloid beta levels ( $\mathrm{ng} / \mathrm{ml}$ ) in control and SAH group rats kept in different environments for 7 days $\left({ }^{* * *} \mathrm{p}<0.001\right)$.
Hippocampus Map2

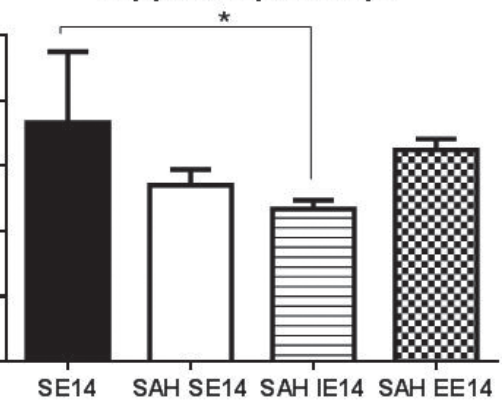

Figure 6: Frontal lobe and hippocampus MAP2 levels ( $\mathrm{ng} / \mathrm{ml}$ ) in control and SAH group rats kept in different environments for 14 days $\left({ }^{*} \mathrm{p}<0.05\right)$.

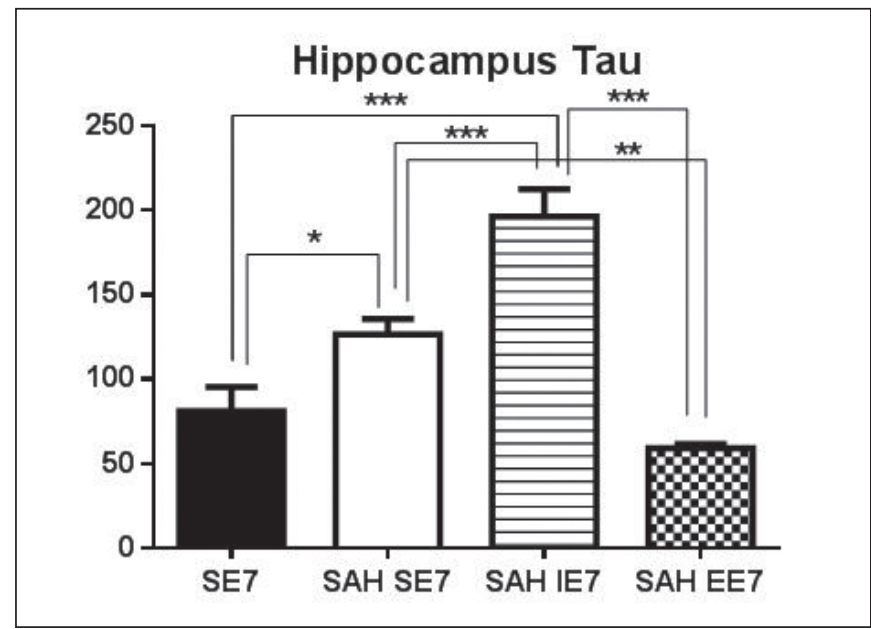

Figure 8: Hippocampus Tau levels (pg/ml) in control and SAH group rats kept in different environments for 7 days $\left({ }^{*} p<0.05\right.$, $\left.{ }^{* *} \mathrm{p}<0.01,{ }^{* * *} \mathrm{p}<0.001\right)$.

Frontal lobe amyloid beta was statistically increased in rats kept in an isolated environment for 7 days compared with frontal lobe amyloid beta in both SAH rats kept in an enriched environment and control rats kept in a standard environment for 7 days (Figure 9).

In the SAH group kept in different environments for 14 days, hippocampal tau and frontal lobe amyloid beta levels were significantly increased in rats kept in an isolated environment. Increasing in hippocampal amyloid beta levels were also statistically significant in rats kept in an isolated environment, compared with rats kept in an enriched environment (Figure 10).

\section{DISCUSSION}

The hippocampus is essential for both forming and replaying memories. The prefrontal cortex involves representations of meaningful contexts in which related memories occur (20). Interactions between the hippocampus and prefrontal cortex are integral to the formation of consciousness and memory (9). In addition, the hippocampus plays an important 


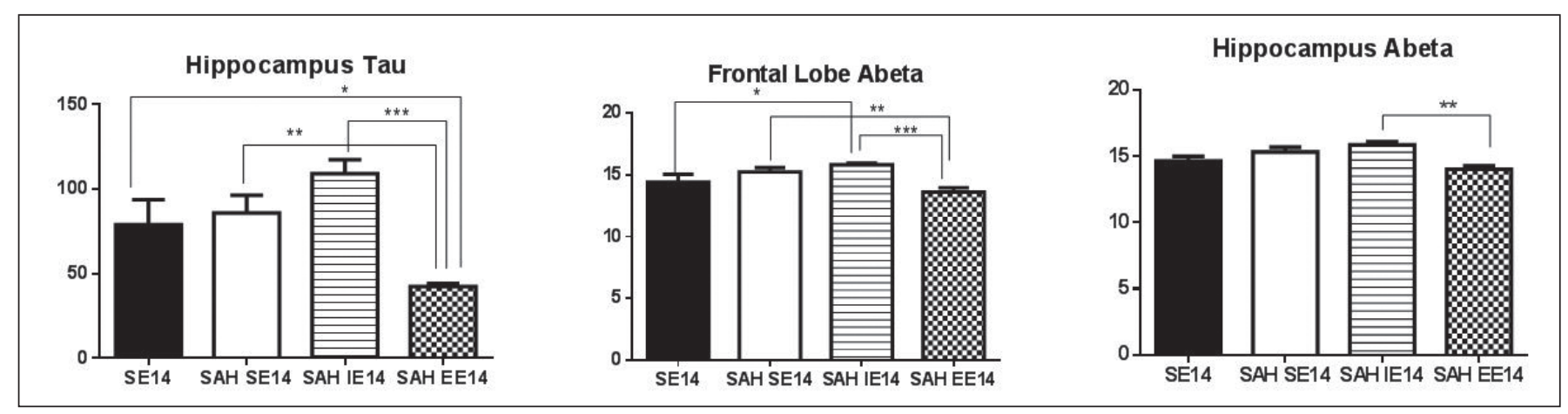

Figure 10: Hippocampus Tau (pg/ml), frontal lobe and hippocampus amyloid beta levels (ng/ml) in control and SAH group rats kept in different environments for 14 days $\left({ }^{*} \mathrm{p}<0.05,{ }^{* *} \mathrm{p}<0.01,{ }^{* \star *} \mathrm{p}<0.001\right)$.

role, particularly in short-term memory, emotion and finding the direction of a target. The frontal cortex is the long-term memory centre, and memory disturbances, lack of awakening and attention may occur when it is damaged $(11,14)$.

Microtubules are major cellular architectural elements and enable neurons to achieve and maintain their shape. They function in many processes including intracellular support and DNA segregation. Neuronal processing is supported by intracellular microtubule integrity and functions $(5,8)$. Tau and other MAPs promote the assembly and stabilisation of neuronal microtubules (5). They regulate microtubule networks and their functions in both axons and dendrites $(4,16)$. MAP proteins are originally characterised by their ability to bind microtubules $(5,7)$. MAPs are divided into two main categories: type I includes MAP1 proteins, and type II includes MAP2, MAP4 and tau proteins. MAP2 are located mostly in dendrites whereas tau is limited to axons $(19,23)$. MAP2 proteins bind a single microtubule to promote stabilisation; tau also facilitates the bundling of microtubules (5).

Reduced MAP2 levels were shown in experimental focal cortical compression, contusion and seizure-related brain damage $(2,10,17)$. In Alzheimer's disease and cerebrovascular disease, tau protein accumulates and amyloid beta fails to be eliminated. Increased amyloid beta also induces both pathological changes in and misfolding of tau proteins. Thus, an interpretation that considers both amyloid beta and tau together would be a more reliable way to evaluate pathological tau protein accumulation in tissue $(3,22,25)$.

Environmental conditions play a dominant role in the basic activity level of the rat brain. Production of brain neurotrophic factors has been shown in rats kept in enriched conditions for the long term. Rich environmental conditions increase neurogenesis and act as nerve cell protectors (13). It has also been shown that an enriched environment increases both learning and memory $(18,21,24)$. A comparative study of the microtubule integrity in SAH and other cerebral pathologies in all three environmental conditions has not yet been published.

In our study, control group frontal lobe MAP2 levels were increased in rats kept in an enriched environment for 7 days.
Frontal lobe and hippocampal amyloid beta levels were increased in rats kept in an isolated environment. Increased MAP2 levels in the hippocampus, decreased frontal and hippocampal amyloid beta were seen in rats kept in an enriched environment for 14 days. After experimental subarachnoid haemorrhage, MAP2 levels in tissues were reduced in rats kept in an isolated environment for both 7 and 14 days. Hippocampal tau and frontal lobe amyloid beta levels were increased in rats kept in an isolated environment for 7 days. Tau proteins in the hippocampus, amyloid beta in both the hippocampus and the frontal lobe were increased in rats kept in an isolated environment for 14 days.

An enriched environment affects both cortical and hippocampal neurons positively $(12,15)$. During the treatment of $\mathrm{SAH}$, keeping the patient in an enriched environment is not well accepted.

\section{CONCLUSION}

It is generally accepted that patients should be in an isolated environment to keep brain oxygen consumption at optimal levels and to maintain normal levels of cerebral perfusion pressure. The present experimental study supports an enriched environmental condition in the follow-up of patients with subarachnoid haemorrhage. We suggest approaching our results cautiously as an enriched environment would be more valuable in the treatment of SAH but may become unnecessarily courageous. This study was adapted for different environments of rat experimental study. In the further studies, environmental conditions may be adjusted according to the intensive care or clinical setting of SAH patients. However, our study may lead to the re-evaluation of ambient conditions during treatment of patients with $\mathrm{SAH}$. Therefore, it is important to note that in discussing this proposal, we believe further experimental studies would producemore reliable results.

\section{ACKNOWLEDGEMENTS}

This study was supported by Scientific Research Project Committee of Eskisehir Osmangazi University (Support 
Number BAP; 201611003). The sponsor had no role in the design or conduct of this research.

\section{- REFERENCES}

1. Al-Khindi T, Macdonald RL, Schweizer TA: Cognitive and functional outcome after aneurysmal subarachnoid hemorrhage. Stroke 41:e519-e536, 2010

2. Ballough GP, Martin LJ, Cann FJ, Graham JS, Smith CD, Kling CE, Forster JS, Phann S, Filbert MG: Microtubule-associated protein 2 (MAP-2): A sensitive marker of seizure-related brain damage. J Neurosci Methods 61(1-2):23-32, 1995

3. Bloom GS: Amyloid- $\beta$ and tau: The trigger and bullet in Alzheimer disease pathogenesis. JAMA Neurol 71(4):505-508, 2014

4. Butner KA, Kirschner MW: Tau protein binds to microtubules through a flexible array of distributed weak sites. J Cell Biol 115:717-730, 1991

5. Conde C, Caceres A: Microtubule assembly, organization and dynamics in axons and dendrites. Nature Reviews Neuroscience 10: 319-332, 2009

6. Craddock TJ, Hameroff SR, Ayoub AT, Klobukowski M, Tuszynski JA: Anesthetics act in quantum channels in brain microtubules to prevent consciousness. Curr Top Med Chem 15(6):523-533, 2015

7. Dehmelt L, Halpain S: The MAP2/TauFamily of Microtubuleassociated proteins. Genom Biol 6(1):204, 2005

8. Dent EW, Baas PW: Microtubules in neurons as information carriers. J Neurochem 129(2):235-239, 2014

9. Franklin DJ, Grossberg S: A neural model of normal and abnormal learning and memory consolidation: Adaptively timed conditioning, hippocampus, amnesia, neurotrophins, and consciousness. Cogn Affect Behav Neurosci 17(1): 2476, 2017

10. Furutani R, Kibayashi K: Morphological alteration and reduction of MAP2-immunoreactivity in pyramidal neurons of cerebral cortex in a rat model of focal cortical compression. J Neurotrauma 29(6):1266-1276, 2012

11. Fuster JM: The prefrontal cortex-an update: Time is of the essence. Neuron 30(2):319-333, 2001

12. Ibi D, Takuma K, Koike H, Mizoguchi H, Tsuritani K, Kuwahara Y, Kamei H, Nagai T, Yoneda Y, Nabeshima T, Yamada K: Social isolation rearing-induced impairment of the hippocampal neurogenesis is associated with deficits in spatial memory and emotion-related behaviors in juvenile mice. J Neurochem 105(3):921-932, 2008
13. Ickes BR, Pham TM, Sanders LA, Albeck DS, Mohammed AH, Granholm AC: Long-term environmental enrichment leads to regional increases in neurotrophin levels in rat brain. Exp Neurol 164(1):45-52, 2000

14. Isaacson RL: Unsolved mysteries: The hippocampus. Behav Cogn Neurosci Rev 1(2):87-107, 2002

15. Jung CK, Herms J: Structural dynamics of dendritic spines are influenced by an environmental enrichment: An in vivo imaging study. Cereb Cortex 24(2): 377-384, 2014

16. Kim H, Binder LI, Rosenbaum JL: The periodic association of MAP2 with brain microtubules in vitro. J Cell Biol 80:266-276, 1979

17. Li GL, Farooque M, Lewen A, Lennmyr F, Holtz A, Olsson Y: MAP2 and neurogranin as markers for dendritic lesions in CNS injury. An immunohistochemical study in the rat. APMIS 108(2):98-106, 2000

18. Li L, Tang BL: Environmental enrichment and neurodegenerative diseases. Biochem Biophys Res Commun 334:293-297, 2005

19. Matus A: Microtubule-associated proteins and neuronal morphogenesis. J Cell Sci Suppl 15:61-67, 1991

20. Preston AR, Eichenbaum $\mathrm{H}$ : Interplay of hippocampus and prefrontal cortex in memory. Curr Biol 23(17):R764-R773, 2013

21. Pryce C, Mohammed A, Feldon J: Environmental manipulations in rodents and primates: Insights into pharmacology, biochemistry and behaviour. Pharmacol Biochem Behav 73:15, 2002

22. Thal D, Attems J, Ewers M: Spreading of amyloid, tau, and microvascular pathology in Alzheimer's disease: Findings from neuropathological and neuroimaging studies. J Alzheimers Dis 42 Suppl 4: 421-429, 2014

23. Tucker RP: The roles of microtubule-associated proteins in brain morphogenesis: A review. Brain Res Rev 15(2):101-120, 1990

24. van Prag $\mathrm{H}$, Kempermann G, Gage FH: Neural consequences of environmental enrichment. Nat Rev Neurosci 1(3):191-198, 2000

25. Weller R, Yow H, Preston S, Mazanti I, Nicoll J: Cerebrovascular disease is a major factor in the failure of elimination of amyloid beta from the aging human brain. Ann N Y Acad Sci 977: 162168,2002 